\title{
Risk of Autoimmune Disease in Adults with Chronic Insomnia Requiring Sleep-Inducing Pills: A Population-Based Longitudinal Study
}

\author{
Victor C. Kok, MMedSc, MD, PhD, FACP ${ }^{1,2}$, Jorng-Tzong Horng, PhD 2,3, Guo-Dung Hung, MD ${ }^{4}$, \\ Jia-Li Xu, BSC ${ }^{3}$, Tzu-Wei Hung, BSC ${ }^{3}$, Yu-Ching Chen, PhD ${ }^{2}$, and Chien-Lung Chen, MD ${ }^{5}$
}

\begin{abstract}
'Department of Internal Medicine, Kuang Tien General Hospital, Taichung, Taiwan; ²Disease Informatics Research Group, Asia University, Taichung, Taiwan; ${ }^{3}$ Department of Computer Science and Information Engineering, National Central University, Jhongli, Taiwan; ${ }^{4}$ Division of Rheumatology \& Immunology, Department of Internal Medicine, Kuang Tien General Hospital, Taichung, Taiwan; ${ }^{5}$ Division of Nephrology, Department of Internal Medicine, Taiwan Landseed Hospital, Taoyuan, Taiwan.
\end{abstract}

\begin{abstract}
BACKGROUND: Recent studies indicate that chronic insomnia is associated with the development of certain somatic diseases. Whether it would be associated with the development of an autoimmune disease (AID) was unknown.
\end{abstract}

OBJECTIVE: We aimed to examine the association and quantify the magnitude of risk for AID in individuals suffering from chronic insomnia requiring sleep-inducing pills.

DESIGN: This was a population-based, nationwide longitudinal study.

PARTICIPANTS: Using a claims data set containing 1 million randomly sampled, insured subjects derived from the National Health Insurance Research Database, we assembled a chronic insomnia group and a 1:3 propensity score-matched comparison group (CP), which were balanced in terms of sex, age, insurance premium, urbanization, alcohol use disorder, smoking-related diagnoses, and morbid obesity.

MAIN MEASURES: Person-time data with incidence rate, adjusted hazard ratios (aHR) by the Cox model, AID-free survival functions compared with the log-rank test, and a sensitivity analysis on the time lag effect were presented. Incident AID within the first year of follow-up were excluded. The error rate was controlled using the BenjaminiHochberg procedure.

KEY RESULTS: With 39,550 and 129,914 person-years' follow-up for the chronic insomnia and CP groups ( $n=5,736$ and 17,208$)$, respectively, we found an increased risk for subsequent AID, representing a $70 \%$ increase in the aHR (1.7; $95 \%$ confidence interval [CI], 1.5-1.9, $p<0.0001)$. A positive association between chronic insomnia and primary Sjögren's syndrome (pSS) was observed (aHR, 1.3; 95 \% CI, 1.1-1.6). Sensitivity analysis disclosed that AID risk was even stronger after 5 years of follow-up (aHR, 2.0; 95 \% CI, 1.7-2.4).

Jia-Li Xu BSc and Tzu-Wei Hung BSc contributed equally to this work.

Electronic supplementary material The online version of this article (doi:10.1007/s11606-016-3717-z) contains supplementary material, which is available to authorized users.

Received December 1, 2015

Revised March 11, 2016

Accepted April 13, 2016

Published online April 29, 2016
CONCLUSION: Chronic insomnia requiring sleepinducing pills may be associated with a $70 \%$ increased risk for future AID, particularly pSS.

KEY WORDS: chronic insomnia; autoimmune disease; population-based study; propensity score matching; Sjögren's syndrome.

J Gen Intern Med 31(9):1019-26

DOI: $10.1007 / \mathrm{s} 11606-016-3717-\mathrm{Z}$

(c) Society of General Internal Medicine 2016

\section{INTRODUCTION}

Sleep is a physiological restorative process that maintains homeostasis in various bodily functions, including the mental, neuro-immunological, cardio-metabolic, and neurocognitive systems. There is a major public health epidemic of sleep disturbance and sleep curtailment worldwide. Insomnia defined as having impaired daytime performance due to difficulty initiating sleep, difficulty maintaining sleep, or early morning awakening without ability to return to sleep despite adequate opportunity and time for sleep has a prevalence of approximately $20 \%$ in an industrialized society. ${ }^{1,2}$ A UK population-based, longitudinal cohort study showed that the prevalence of insomnia in the adult UK general population is $37 \%$, and that much of this insomnia is persistent, with over two-thirds (69\%) of these individuals also reporting symptoms a year later. ${ }^{3}$ A survey conducted in 2005 in Taiwan demonstrated that one-fourth of Taiwanese adults experienced insomnia, with the most prevalent symptoms being difficulty initiating sleep in $15 \%$ of insomniacs, early morning awakening in $14 \%$ and difficulty maintaining sleep in $13 \%{ }^{4}$ Chronic insomnia, defined as unsatisfactory in either sleep quantity or quality causing clinically significant distress or impairment in daily living or functioning occurring at least 3 nights a week for $\geq 3$ months, has a prevalence of $10 \%$ in the general population. ${ }^{1}$

Several epidemiologic studies have reported positive associations between chronic insomnia and various subsequent medical conditions such as cancer, metabolic diseases, cerebral stroke, and heart failure, which suggests that chronic insomnia may be a risk factor or medical condition that 
precedes the occurrence of certain diseases. ${ }^{5-11}$ A large population-based Norwegian study suggested that after adjusting for confounding factors, insomnia remained a significant risk factor for rheumatic diseases, including fibromyalgia (odds ratio [OR], 2.05; $95 \%$ confidence interval [CI], 1.51-2.79) and rheumatoid arthritis (OR, 1.87; $95 \%$ CI, 1.29 2.52). ${ }^{8}$ However, there is no systemic longitudinal evidence linking chronic insomnia with autoimmune diseases (AID).

The finding that dendritic cells linked to the development of AID can be modulated by the restorative process of sleep provides a mechanistic rationale for studying the association between chronic insomnia and subsequent AID. ${ }^{12,13}$

Dysfunctional and defective binding of self-molecules to toll-like receptor (TLR) and inadequately activated dendritic cells have been implicated in the development of AID, while monocyte-derived dendritic cells have been confirmed in the pathogenesis of Sjögren's syndrome. ${ }^{12}$ Research evidence indicates that sleep can effectively enhance adaptive immune responses through the precursors of interleukin-12-producing myeloid dendritic cells. ${ }^{13,14}$ These intertwined findings, centered upon myeloid dendritic cells as the pathogenetic basis of AID, provide us with clues and the rationale to link chronic insomnia with the development of AID in future. Therefore, we conducted this longitudinal study to test the hypothesis of this association.

\section{MATERIALS AND METHODS}

\section{Study Design}

We designed a population-based cohort study in Taiwan with 1:3 propensity score matching for the comparison group, with the longest follow-up being 12 years. The study was performed after obtaining approval from the Kuang Tien General Hospital Institutional Review Board (approval certificate: KTGH IRB-10428), and was conducted according to the Declaration of Helsinki.

Data Source. The National Health Insurance Research Database (NHIRD), a population-based and nationwide medical claims database, was adopted for the research. The analyses included a NHIRD-derived data set, the Longitudinal Health Insurance Database, with 1 million randomly recruited, insured individuals from the mother NHIRD database. We targeted the study population that had medical claim records from 1996 to 2008. The data set was de-identified on the levels of all insured participants and medical institutions prior to release for academic research purposes. The authors have previously conducted several population-based longitudinal studies using the NHIRD-derived data set on clinical epidemiology with study design and methodology similar to the current one. ${ }^{15-19}$

Target Population. The target population for this work was individuals aged 20 years or older with chronic insomnia taking sleep-inducing pills of their physician's choice without any preexisting AID before the index date. The index date was defined as the date of accrual of the cases and the corresponding date for the comparators they were matched to. The beginning year 1996 in the data set was used as a wash-out period so that patients with pre-existing AID could be detected and excluded from the construction of the cohort. The exclusion criteria included records with missing data, age $<20$ years, patients with a preexisting AID before the index date, individuals never prescribed with any hypnotics, and having $<2$ medical claims over the first 3-month period. The exclusion criterion for the comparison group was subjects subsequently prescribed hypnotics during follow-up. The comparison group was formed after 1:3 propensity score matching using the perfect matching algorithm by sex, age, index date, smoking-related diagnoses, alcohol use disorder, morbid obesity, household insurance premium, and urbanization level (Fig. 1). The household insurance premiums of the participants for the index year were used as a proxy for household income, based on the fact that payroll-based premiums paid to the National Health Insurance are proportional to household income level. ${ }^{20}$

Diagnosis Criteria. According to the Diagnostic and Statistical Manual of Mental Disorders, fourth ed. (DSM-IV) classification for insomnia (International Classification of Disease, ninth edition, with clinical modifications, ICD-9$\mathrm{CM}$, code 780.52), the diagnostic criteria of chronic insomnia in adults is a predominant complaint of dissatisfaction with sleep quality or quantity, not attributable to the physiological effects of a substance, occurring at least 3 nights a week for 3 months or more, associated with at least one of the following symptoms: difficulty initiating sleep, difficulty maintaining sleep characterized by frequent awakenings or problems returning to sleep after awakenings, and early morning awakening with inability to return to sleep. Furthermore, this insomnia must have caused clinically significant distress or impairment in personal or social functioning in daily living. Individuals with chronic insomnia qualified for this study if they had physician-diagnosed insomnia and a prescription of sleep-inducing pills in two independent medical claims using the ICD-9-CM code 780.52 within a 3-month period. The index date was the last day of the third month observed.

Outcome Measures. The study endpoint was the development of a physician-diagnosed AID. In this study, the investigated AID and corresponding ICD-9-CM code(s) were: primary Sjögren's syndrome (pSS; 710.2) without concurrent codes of any other AID; systemic lupus erythematosus (710.0); ankylosing spondylitis (720); polymyositis/dermatomyositis (710.3 and 710.4); multiple sclerosis (340); vasculitis (including hypersensitivity angiitis and anti-glomerular basement membrane antibody-mediated disease, 446.2 and 446.29); rheumatoid arthritis (714); Behçet's disease (136.1); myasthenia gravis (358); systemic sclerosis (710.1); Guillain-Barré syndrome (357.0); Hashimoto's thyroiditis (245.2); Graves' 


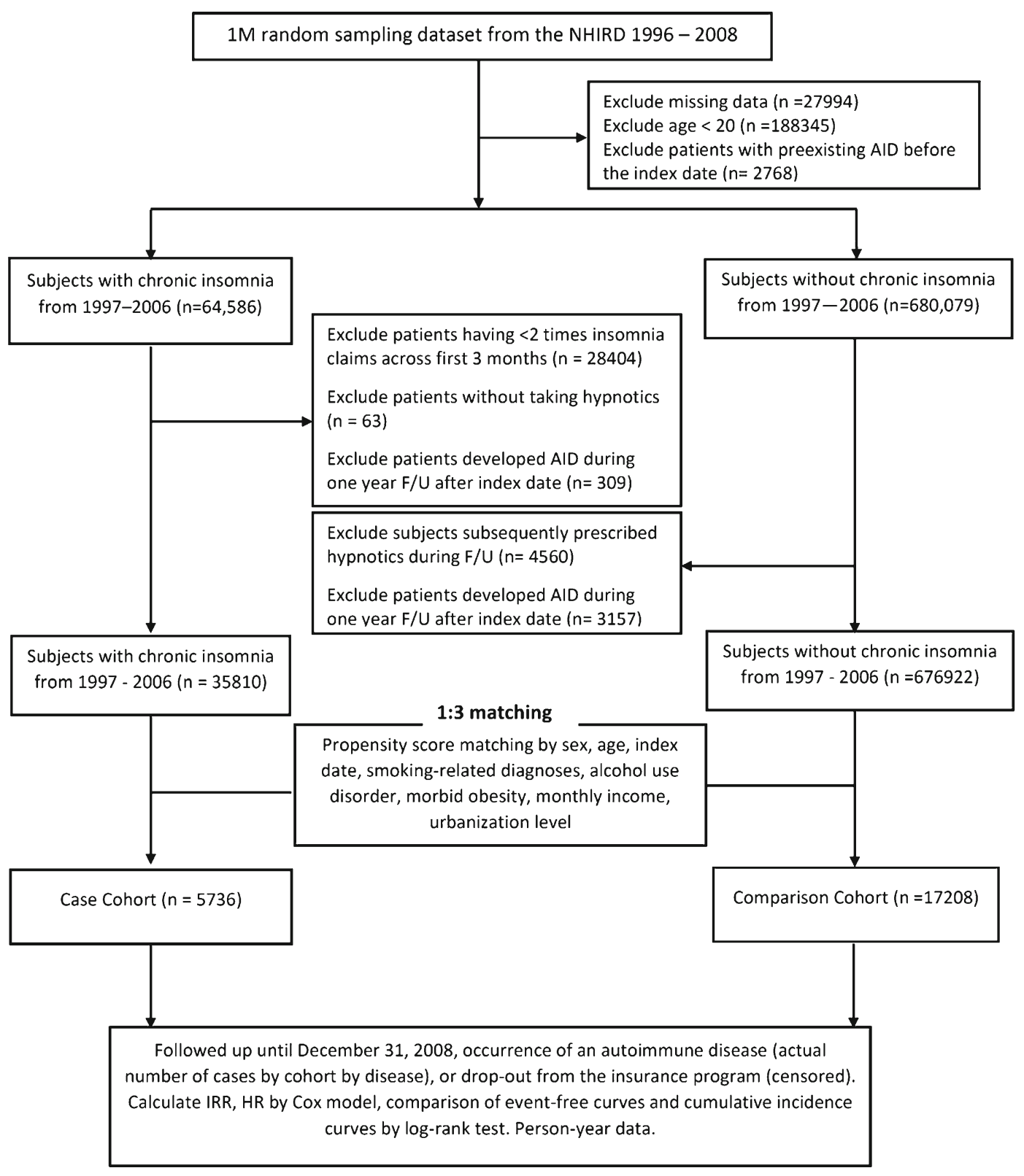

Figure 1. CONSORT diagram of the study flow.

disease (242 and 242.01), and uveitis (364.00 and 364.01). Three repeated claims with one of the above ICD-9-CM codes were required to confirm the presence of the outcome. The incidence of AID with person-year data are presented in the results. Figure 1 reviews the step-by-step process in the assembly of the case and the comparison cohorts and their follow-up.

Statistical Analysis. We used the SAS statistical package (SAS System for Windows, version 9.4; SAS Institute, Cary, NC, USA) to conduct all the statistical analyses required in this work. In this study, each patient $(n=22,944)$ was individually tracked for up to 12 years from their index date to identify those who were subsequently diagnosed as having AID. The age-, demographic-, and comorbidity-specific incidence density rates of AID were calculated in each subgroup in person-years. Both univariate and multivariate Cox proportional hazards regression models were conducted at $\alpha=0.05$ significance level to calculate the crude hazard ratio (HR) and mutually adjusted HR with their $95 \% \mathrm{CI}$ after controlling for all the selected sociodemographic and comorbidity variables. To satisfy the assumption of non-informative censoring, censoring of individual subjects was not related to the probability of occurrence of an event occurring; for example, continuation of follow-up in the cohort did not depend on a subject's medical condition. We checked the survival curves in SAS using Proc Lifetest to ascertain that the hazard functions were proportional over time. We graphed $\log (-\log [$ survival] $)$ versus the log of the survival time to see if they resulted in parallel curves if the predictor was proportional. A table of the measured HRs for each type of AID using the multivariate Cox models was constructed after controlling for sex, age group, 
household insurance premium, urbanization level, smokingrelated diagnoses, diabetes mellitus (DM), chronic kidney disease (CKD), liver cirrhosis, and depression. The KaplanMeier method and log-rank test were subsequently applied to examine the differences of the cumulative incidence for the AID between the chronic insomnia group and the comparison group. To control for type I errors, the Benjamini-Hochberg $\mathrm{BH}$ ) procedure was implemented to achieve false discovery rate (FDR) control. ${ }^{21}$ In short, the $\mathrm{BH}$ procedure is used to determine which $p$ values to declare statistically significant when the $p$ value for an individual hypothesis test is $\leq d x \mathrm{i} / \mathrm{n}$, where $d$ represents the maximal FDR set as $10 \%$ wherein case validation in the subsequent study procedure can be performed; $i$ means the test of rank 1, 2, through $i$ in ascending order of sorted $p$ values; and $n$ equals the total number of hypothesis tests, which was 15 in this work. ${ }^{22}$

\section{RESULTS}

After a total of 169,463 person-years' follow-up $(39,550$ person-years for the chronic insomnia group and 129,914 person-years for the comparison group) of the entire cohort, the chronic insomnia group that required sleep-inducing pills had a 1.8-fold increase in AID in terms of the incidence density rate as compared to people without chronic insomnia; moreover, there appeared to be an increased risk for a subsequent AID, representing a $70 \%$ increase in the aHR $(1.7 ; 95 \%$ CI, $1.5-1.9 ; p<0.0001)$ after controlling for age, sex, household insurance premium, urbanization, alcohol- and smokingrelated diagnoses, morbid obesity, and comorbidities. Figure 2 demonstrates the curves of the cumulative incidence of AID over the follow-up period of the two compared groups. The log-rank test for the survival functions showed high statistical significance $(p<0.0001)$.
There were 5736 participants in the chronic insomnia group and 17,208 in the comparison group; $52 \%$ of the participants were male. It is notable that $66.5 \%$ of the insomniac subjects were aged 20 to 49 years. Eighty-five percent of the chronic insomnia group was recruited from the two lowest categories of income, indicating that the insomniac patients were more likely to be socioeconomically disadvantaged than the comparison subjects. Sixty-six percent of the subjects in this study lived in an urban area (Table 1).

Slightly more subjects in the chronic insomnia group had $\operatorname{DM}(6.14$ vs $3.42 \%)$, depression (5.58 vs $1.88 \%$ ), CKD (1.17 vs $0.53 \%$ ), and liver cirrhosis ( 0.71 vs $0.32 \%$ ) (Table 1$)$.

Table 2 depicts the incidence of AID under specific personyears' follow-up and analysis showing the univariate and multivariate aHRs as stratified by outcome, age group, sex, socioeconomic proxy, geographic urbanization level, smokingrelated diagnoses, and comorbidities such as DM, CKD, liver cirrhosis, and depression. Compared with males, females had a statistically significant $70 \%$ increased risk for AID, with an aHR of 1.7 (95\% CI, 1.4-2.1; $p<0.0001)$. Patients with DM and depression had increased risk for AID, with similar aHRs and $95 \% \mathrm{CI}$ of 1.4 (95\% CI, 1.0-2.0) (Table 2).

Among the various types of AID, pSS alone had a statistically significant association with chronic insomnia, and the results indicate that as compared with the population without chronic insomnia, the individuals with chronic insomnia had a $30 \%$ increased risk of pSS (aHR, 1.3; $95 \%$ CI, 1.1-1.6; $p=0.0073$ ) (Table 3). The null hypothesis in this setting was therefore rejected, which was subsequently verified under FDR control using the BH procedure (Online Supplemental Table 1).

Sensitivity analysis was performed to assess the risk of AID over a longer follow-up period, which showed that AID risk was even stronger after 5 years of follow-up, with an aHR of 2.0 (95\% CI, 1.7-2.4) (Table 4).

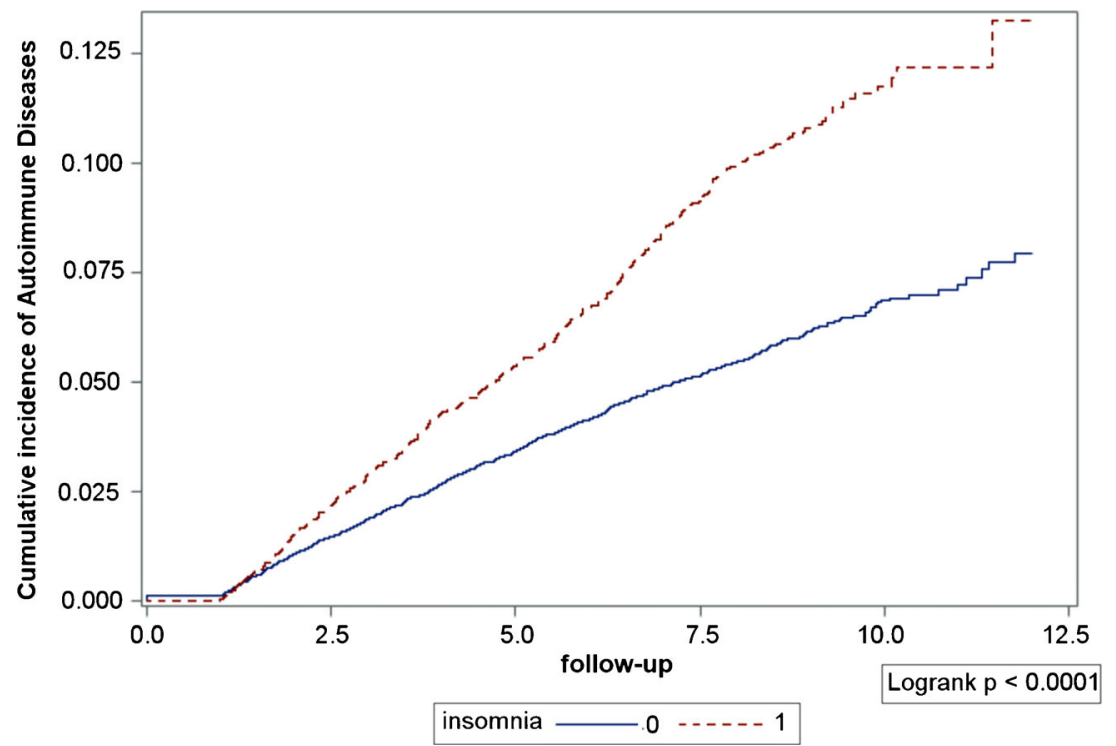

Figure 2. Cumulative incidence curves of autoimmune disease in the chronic insomnia group and the comparison group as compared by a log-rank test. 
Table 1. Demographics of the Studied Cohorts Matched by Propensity Score on Sex, Age, Index Date, Socioeconomic Status Proxy, Alcohol Use Disorder, Smoking-Related Diagnoses, and Morbid Obesity

\begin{tabular}{lcc}
\hline \hline & $\begin{array}{l}\text { Insomnia } \\
\boldsymbol{n}=\mathbf{5 7 3 6}(\mathbf{\%})\end{array}$ & $\begin{array}{l}\text { Non-insomnia } \\
\boldsymbol{n}=\mathbf{1 7 , 2 0 8}(\mathbf{\%})\end{array}$ \\
\hline Sex & & \\
$\quad$ Male & $3010(52.48)$ & $9030(52.48)$ \\
Female & $2726(47.52)$ & $8178(47.52)$ \\
Age (years) & $961(16.75)$ & $2883(16.75)$ \\
20-29 & $1278(22.28)$ & $3834(22.28)$ \\
$30-39$ & $1576(27.48)$ & $4728(27.48)$ \\
$40-49$ & $991(17.28)$ & $2973(17.28)$ \\
$50-59$ & $587(10.23)$ & $1761(10.23)$ \\
60-69 & $288(5.02)$ & $864(5.02)$ \\
$70-79$ & $55(0.96)$ & $165(0.96)$ \\
$80-89$ & 0 & 0 \\
90-99 & $1994(34.76)$ & $5982(34.76)$ \\
Household insurance premium & $2854(49.76)$ & $8562(49.76)$ \\
$\leq$ NT 15,840 & $888(15.48)$ & $2664(15.48)$ \\
15,841-25,000 & & \\
$\geq$ NT 25,001 & $3788(66.04)$ & $11,364(66.04)$ \\
Urbanization level & $1665(29.03)$ & $4995(29.03)$ \\
Urban & $283(4.93)$ & $849(4.93)$ \\
Suburban & $2(0.03)$ & $6(0.03)$ \\
Rural & $97(1.69)$ & $291(1.69)$ \\
Alcohol use disorder & 0 & 0 \\
Smoking-related diagnoses & & \\
Morbid obesity & $352(6.14)$ & $591(3.42)$ \\
Comorbidities & $320(5.58)$ & $323(1.88)$ \\
Diabetes mellitus & $67(1.17)$ & $91(0.53)$ \\
Depression & $41(0.71)$ & $55(0.32)$ \\
Chronic kidney disease & & \\
Liver cirrhosis & & \\
\hline
\end{tabular}

Confirmation and validation of all cases of pSS were carried out by checking each individual's medical claims record on information such as the median age at diagnosis, if they had ever undergone a sialoscintigraphy or salivary gland biopsy, if they had ever had anti-SSA/Ro and/or anti$\mathrm{SSB} / \mathrm{La}$ serologic tests, and subsequent treatment with hydroxychloroquine. Online Supplemental Table 2 demonstrates that the median age at diagnosis of pSS was 52 and 54 years in the chronic insomnia group and the comparison group, respectively. All patients with pSS in the chronic insomnia group had undergone sialoscintigraphy, whereas this rate was nearly $98 \%$ in the comparison group. All but one patient with pSS had undergone at least one serologic test of anti-SSA/Ro or anti-SSB/La according to their claims record. These data provide convincing evidence that the diagnosis of pSS was reliable in this data set. In Taiwan, this study also demonstrated that approximately $60 \%$ of the patients with pSS were treated with hydroxychloroquine, which is normally indicated for the management of extraglandular manifestations such as fatigue, arthralgia, and myalgia.

\section{DISCUSSION}

For the first time, to the best of our knowledge, this population-based longitudinal study with up to 12 years of follow-up confirms an association between chronic insomnia and a resultant acquired AID, primarily pSS, in the future. The magnitude of the risk of pSS in subjects with chronic insomnia, although modest, with an aHR of 1.3, represents a $30 \%$ statistically significant increase when compared to subjects without insomnia. Although the detailed mechanism is still unclear, the results support our research hypothesis and the working hypothesis that the restorative effect of sleep is important in the neuro-immunologic function of an individual. ${ }^{13,14,23}$

pSS, also called sicca syndrome, is a chronic AID in which the lacrimal and salivary glands undergo progressive destruction by lymphocytes and plasma cells, resulting in both keratoconjunctivitis sicca (dry eyes) and xerostomia (feeling of dry mouth). The prevalence of pSS in East Asian Chinese was estimated to be $0.77 \%$ by the Copenhagen criteria and $0.33 \%$ by the San Diego criteria in a study of Beijing (Peking) residents. ${ }^{24}$ In agreement with the literature, the median age of our patients at pSS diagnosis was approximately 5254 years. It is noteworthy that 56 to $60 \%$ of patients with pSS in Taiwan are treated with hydroxychloroquine, which is commonly prescribed by rheumatologists in Taiwan to manage extraglandular manifestations such as fatigue, arthralgia, and myalgia.

Study design and case ascertainment are two of the most crucial steps to reach solid conclusions in any populationbased longitudinal study. Our study employed propensity score matching by sex, age, index date, smoking- and alcohol-related diagnoses, morbid obesity, and socioeconomic proxies such as monthly income and urbanization level to assemble the comparison group in order to achieve a similar level of comparison to a prospective controlled study. In terms of case ascertainment, we defined chronic insomnia as the state of requiring sleep-inducing medication; further, in the subsequent analysis, we checked each individual who developed pSS in terms of their clinical investigations using sialoscintigraphy and anti-SSA/Ro and anti-SSB/La immunologic serologic tests, the results of which demonstrated that almost all of them had undergone these examinations, indicating a high level of validation. To help clarify the issue of temporality of the primary association and account for the biological onset of undiagnosed AIDs, we implemented a sensitivity analysis for unmeasured confounding due to undiagnosed AIDs by imposing a lag time for the effect of chronic insomnia on the AID. Incident AID within the first year of follow-up were excluded from the analysis to avoid protopathic bias (reverse causation); in addition, lag time analyses were performed by varying the lag time (from exposure to outcome) to check if the association between chronic insomnia and the AID remained statistically significant in the subsequent sensitivity analysis. We believe that we have provided ample evidence for this positive association between chronic insomnia and AID.

There were higher rates of medical comorbidities in the chronic insomniacs than in the comparison group in our study, including DM (6.14 vs. $3.42 \%)$, depression (5.58 
Table 2. Incidence of Autoimmune Diseases (AID), Person-Time Rate, and Stratified Analysis Showing Hazard Ratios (HR) from a Multivariate Cox Regression Model

\begin{tabular}{|c|c|c|c|c|c|}
\hline Variable & AID & PYs & Rate & Crude HR (95 \% CI) & Adjusted HR (95\% CI) \\
\hline \multicolumn{6}{|l|}{ Chronic insomnia } \\
\hline No $(n=17,208)$ & 860 & $129,913.55$ & 66.20 & Ref & Ref \\
\hline Yes $(n=5736)$ & 482 & $39,549.79$ & 121.87 & $1.7(1.6-2.0)$ & $1.7(1.5-1.9)^{* *}$ \\
\hline \multicolumn{6}{|l|}{ Age group (years) } \\
\hline $20-39(n=2239)$ & 174 & $14,624.7$ & 118.98 & $0.8(0.3-1.7)$ & $0.8(0.3-1.8)$ \\
\hline $40-59(n=2567)$ & 210 & $18,157.39$ & 115.66 & $0.8(0.3-1.8)$ & $0.6(0.3-1.4)$ \\
\hline $60-79(n=875)$ & 92 & 6361.41 & 144.62 & $1.0(0.4-2.2)$ & $1.1(0.4-2.2)$ \\
\hline $80-89(n=55)$ & 6 & 406.29 & 147.68 & Ref & Ref \\
\hline \multicolumn{6}{|l|}{ Sex } \\
\hline Male $(n=3010)$ & 185 & $19,105.47$ & 96.83 & Ref & Ref \\
\hline Female $(n=2726)$ & 297 & $20,444.32$ & 145.27 & $0.6(0.5-0.7)$ & $1.7(1.4-2.1)^{* *}$ \\
\hline \multicolumn{6}{|l|}{ Household insurance premium } \\
\hline$\leq \mathrm{NT} 15,840(n=1994)$ & 154 & $12,861.97$ & 119.73 & Ref & Ref \\
\hline $15,841-25,000(n=2854)$ & 249 & $20,199.68$ & 123.27 & $1.0(0.8-1.2)$ & $1.0(0.8-1.2)$ \\
\hline$\geq$ NT $25,001 \quad(n=888)$ & 79 & 6488.15 & 121.76 & $1.0(0.8-1.3)$ & $1.1(0.8-1.4)$ \\
\hline \multicolumn{6}{|l|}{ Urbanization level } \\
\hline Rural $(n=283)$ & 27 & 2164.35 & 124.75 & Ref & Ref \\
\hline Suburban $(n=1665)$ & 130 & $12,008.87$ & 108.25 & $0.9(0.6-1.3)$ & $0.9(0.6-1.4)$ \\
\hline Urban $(n=3788)$ & 325 & $25,376.57$ & 128.08 & $1.0(0.7-1.5)$ & $1.1(0.7-1.7)$ \\
\hline Alcohol use disorder & 0 & - & - & - & - \\
\hline Smoking-related diagnoses & 7 & 531.97 & 131.59 & $1.9(1.1-3.2)$ & $1.6(0.9-2.8)$ \\
\hline Morbid obesity & 0 & - & - & - & - \\
\hline \multicolumn{6}{|l|}{ Comorbidities } \\
\hline \multicolumn{6}{|l|}{ Diabetes mellitus } \\
\hline No $(n=5384)$ & 463 & $37,668.87$ & 122.91 & Ref & Ref \\
\hline Yes $(n=352)$ & 19 & 1880.93 & 101.01 & $1.5(1.1-2.1)$ & $1.4(1.0-2.0)^{*}$ \\
\hline \multicolumn{6}{|l|}{ Chronic kidney disease } \\
\hline No $(n=5669)$ & 478 & $39,183.90$ & 121.99 & Ref & Ref \\
\hline Yes $(n=67)$ & 4 & 365.89 & 109.32 & $1.4(0.6-2.9)$ & $1.2(0.6-2.5)$ \\
\hline \multicolumn{6}{|l|}{ Liver cirrhosis } \\
\hline No $(n=5695)$ & 478 & $39,322.52$ & 121.59 & Ref & Ref \\
\hline Yes $(n=41)$ & 4 & 227.28 & 176.00 & $1.5(0.6-3.8)$ & $1.6(0.7-3.9)$ \\
\hline \multicolumn{6}{|l|}{ Depression } \\
\hline No $(n=5416)$ & 460 & $37,898.19$ & 121.38 & Ref & Ref \\
\hline Yes $(n=320)$ & 22 & 1651.60 & 133.20 & $1.5(1.0-2.1)$ & $1.4(1.0-2.0)^{*}$ \\
\hline
\end{tabular}

CI confidence interval, PYs person-years, rate incidence rate, per 10,000 person-years, Ref reference Adjusted model was mutually adjusted. $* p<0.05 ; * p<0.0001$

vs. $1.88 \%)$, CKD (1.17 vs. $0.53 \%)$, and liver cirrhosis (0.71 vs. $0.32 \%)$. These observations are supported by research findings in the literature. ${ }^{25}$ In this large community-based cross-sectional study, the odds of insomnia was higher in people with diabetes (OR, 1.4; $95 \% \mathrm{CI}$, $1.05-2.0 ; p=0.04){ }^{25}$

Table 3. Measured Hazard Ratios (HR) for Selected Types of Autoimmune Disease (AID) from a Multivariate Cox Proportional Hazards Regression Model

\begin{tabular}{|c|c|c|c|}
\hline Type of AID $(x / y)^{a}$ & $\begin{array}{l}\text { Comparison group } \\
\text { reference }\end{array}$ & $\begin{array}{l}\text { Chronic insomnia group } \\
\text { HR (95\% CI) }\end{array}$ & $p$ value \\
\hline All types of AID $(860 / 482)$ & 1 & $1.7(1.5-1.6)$ & $<0.0001$ \\
\hline Primary Sjögren's syndrome $(212 / 164)$ & 1 & $1.3(1.1-1.6)$ & 0.0073 \\
\hline $\operatorname{SLE}(39 / 29)$ & 1 & $1.4(0.9-2.3)$ & 0.1432 \\
\hline Ankylosing spondylitis $(5 / 6)$ & 1 & $2.0(0.6-6.6)$ & 0.2574 \\
\hline Polymyositis/dermatomyositis $(31 / 23)$ & 1 & $1.2(0.7-2.1)$ & 0.4703 \\
\hline Multiple sclerosis $(6 / 5)$ & 1 & $1.5(0.4-5.1)$ & 0.5044 \\
\hline Vasculitis $^{\text {b }}(3 / 3)$ & 1 & $1.3(0.2-7.0)$ & 0.7436 \\
\hline Rheumatoid arthritis (14/9) & 1 & $1.1(0.5-2.5)$ & 0.8542 \\
\hline Behçet's disease $(8 / 5)$ & 1 & $1.0(0.3-3.0)$ & 0.9677 \\
\hline Myasthenia gravis $(3 / 2)$ & 1 & $0.9(0.1-5.5)$ & 0.9139 \\
\hline Systemic sclerosis $(2 / 1)$ & 1 & $0.7(0.1-9.4)$ & 0.8074 \\
\hline Guillain-Barré syndrome (14/7) & 1 & $0.9(0.3-2.1)$ & 0.7365 \\
\hline Hashimoto's thyroiditis $(33 / 14)$ & 1 & $0.8(0.4-1.5)$ & 0.4637 \\
\hline Graves' disease $(50 / 23)$ & 1 & $0.8(0.5-1.2)$ & 0.2650 \\
\hline Uveitis $(108 / 46)$ & 1 & $0.7(0.5-1.0)$ & 0.0341 \\
\hline
\end{tabular}

Model adjusted for sex, age group, household insurance premium, urbanization level, smoking-related diagnoses, diabetes mellitus, chronic kidney disease, liver cirrhosis, and depression

${ }^{a}(x / y)$ : number of AID in the comparison cohort/number of AID in the insomnia cohort. A participant could have more than one subtype of AID except for primary Sjögren's syndrome

${ }^{b}$ Vasculitis includes hypersensitivity angiitis and anti-GBM antibody-mediated disease

CI confidence interval, SLE systemic lupus erythematosus 
Table 4. Sensitivity Analysis on the Time Lag Effect on the Adjusted Hazard Ratios (HRs) for Subsequent AID in the Chronic Insomnia Cohort

\begin{tabular}{|c|c|c|c|c|}
\hline Subsequent years of follow-up & $\begin{array}{l}\text { Incidence of AID in the } \\
\text { comparison cohort }\end{array}$ & $\begin{array}{l}\text { Incidence of AID in the } \\
\text { insomnia cohort }\end{array}$ & $\begin{array}{l}\text { Adjusted HRs } \\
(95 \% \text { CI) }\end{array}$ & $\overline{p \text { value }}$ \\
\hline 2nd year and beyond & 860 & 482 & $1.7(1.5-1.9)$ & $<0.0001$ \\
\hline 3 rd year and beyond & 676 & 393 & $1.8(1.6-2.0)$ & $<0.0001$ \\
\hline 4th year and beyond & 543 & 319 & $1.8(1.6-2.1)$ & $<0.0001$ \\
\hline 5th year and beyond & 416 & 247 & $1.9(1.6-2.2)$ & $<0.0001$ \\
\hline 6th year and beyond & 308 & 196 & $2.0(1.7-2.4)$ & $<0.0001$ \\
\hline
\end{tabular}

Cox model adjusted for sex, age, household insurance premium, urbanization level, diabetes mellitus, and depression Incident AID within the first year of follow-up were excluded from analysis to avoid protopathic bias

A previous epidemiologic cohort study linked insomnia with self-reported rheumatoid arthritis in a large population-based prospective study in Norwegian people, which showed an OR of 1.87 (95\% CI, 1.29-2.52). ${ }^{8}$ The data from our longitudinal study does not support this association. The main difference between the Norwegian and our Taiwanese study was the definition of the outcome measure: our study used physician-diagnosed rheumatoid arthritis as the outcome measure, which we believe is more reliable than self-reporting.

The finding suggesting that chronic insomnia is a risk factor for pSS is surprising. In addition to the dendritic cells hypothesis stated in the Introduction, ${ }^{12,13,26}$ chronic insomnia is likely to affect the regulation of signaling pathways related to the immune system. ${ }^{27}$ Further research including molecular epidemiology and prospective studies are necessary to confirm whether chronic insomnia is a risk factor for pSS.

We acknowledge some potential limitations to the present study. We did not have access to the relevant biochemistry and serologic test results, nor family history of AID and personal lifestyle histories such as occupation, daily habitual activities, and records of physical activity. Polysomnography and chin or limb electromyography data of the patients were also not available. This is mainly because the personal medical records of the patients were not available, owing to the deidentification of the individuals within the data set. The potential impact of lacking these modifying confounders in Cox models on the risk of AID, although unclear, cannot be large enough to change the direction of the overall risk estimation. Finally, another potential limitation is the lack of covariate analyses of medication use, particularly the type of sleepinducing pills prescribed and concurrent medications taken, which were too complex to be incorporated into this study. It is not possible to ascertain whether the patients actually took the medications prescribed to them. The main purpose of accruing only those who were taking hypnotics into the chronic insomnia group was to validate the physician's diagnosis and to avoid misclassification bias. In the real world, a high proportion of insomniacs particularly those with difficulty in maintaining sleep do not do well despite the hypnotics use. Many have self-medication tendencies by self-administering their prescribed hypnotics in the middle of the night after nocturnal awakenings. According to a population-based study by Roth $\mathrm{T}$ et al. one-fifth of patients with prescription hypnotics readministered another dose in the middle of the night. ${ }^{28}$

\section{CONCLUSIONS}

Chronic insomnia requiring sleep-inducing pills in this East Asian population-based longitudinal study may be associated with a $70 \%$ increased risk for a future AID, particularly $\mathrm{pSS}$. Although this is the first report to demonstrate this positive association, the efficient study design and robust statistical analyses of our study support this finding. Before further evidence surfaces, clinicians should maintain a high level of suspicion to achieve early diagnosis of pSS in individuals afflicted with chronic insomnia.

Acknowledgments: The authors are grateful to the National Health Insurance Administration, Ministry of Health and Welfare, and the National Health Research Institute, both of Taiwan, for kindly providing access to the research data for this study. The interpretation and conclusions contained herein do not represent those of the aforementioned institutions. The authors would like to thank Enago (www.enago.com) for the English language review.

Corresponding Author: Victor C. Kok, MMedSc, MD, PhD, FACP; Department of Internal Medicine, Kuang Tien General Hospital, 117 Shatien Road, Taichung 43303, Taiwan (e-mail: victorkok@asia.edu.tw).

\section{Compliance with Ethical Standards:}

Conflict of Interest Statement: The authors declare that they do not have a conflict of interest.

Funding: This work has no specific fundings.

\section{REFERENCES}

1. Buysse DJ. Insomnia JAMA. 2013;309(7):706-716. doi:10.1001/jama. 2013.193.

2. Riemann D, Spiegelhalder K, Espie C, Pollmacher T, Leger D, Bassetti C, et al. Chronic insomnia: clinical and research challenges-an agenda. Pharmacopsychiatry. 2011;44(1):1-14. doi:10.1055/s-0030-1267978.

3. Morphy H, Dunn KM, Lewis M, Boardman HF, Croft PR. Epidemiology of insomnia: a longitudinal study in a UK population. Sleep. 2007;30(3):274-280.

4. Kao CC, Huang CJ, Wang MY, Tsai PS. Insomnia: prevalence and its impact on excessive daytime sleepiness and psychological well-being in the adult Taiwanese population. Qual Life Res Int $\mathrm{J}$ Qual Life Asp Treat Care Rehab. 2008;17(8):1073-1080. doi:10.1007/s11136-0089383-9.

5. Carroll JE, Irwin MR, Stein Merkin S, Seeman TE. Sleep and multisystem biological risk: a population-based study. PLoS One. 2015;10(2):e0118467. doi:10.1371/journal.pone.0118467. 
6. Wu MP, Lin HJ, Weng SF, Ho CH, Wang JJ, Hsu YW. Insomnia subtypes and the subsequent risks of stroke: report from a nationally representative cohort. Stroke. 2014;45(5):1349-1354. doi:10.1161/STROKEAHA.113. 003675.

7. Sofi F, Cesari F, Casini A, Macchi C, Abbate R, Gensini GF. Insomnia and risk of cardiovascular disease: a meta-analysis. Eur J Prev Cardiol. 2014;21(1):57-64. doi:10.1177/2047487312460020.

8. Sivertsen B, Lallukka T, Salo P, Pallesen S, Hysing M, Krokstad S, et al. Insomnia as a risk factor for ill health: results from the large populationbased prospective HUNT Study in Norway. J Sleep Res. 2014;23(2):124132. doi: $10.1111 /$ jsr. 12102 .

9. Tofield A. Insomnia linked to increased risk of heart failure. Eur Heart J. 2013;34(24): 1771.

10. Vgontzas AN, Liao D, Pejovic S, Calhoun S, Karataraki M, Bixler EO. Insomnia with objective short sleep duration is associated with type 2 diabetes: A population-based study. Diabetes Care. 2009;32(11):19801985. doi: $10.2337 /$ dc09-0284.

11. Vgontzas AN, Liao D, Bixler EO, Chrousos GP, Vela-Bueno A. Insomnia with objective short sleep duration is associated with a high risk for hypertension. Sleep. 2009;32(4):491-497.

12. Vogelsang P, Karlsen M, Brun JG, Jonsson R, Appel S. Altered phenotype and Statl expression in Toll-like receptor $7 / 8$ stimulated monocyte-derived dendritic cells from patients with primary Sjogren's syndrome. Arthritis Res Ther. 2014;16(4):R166. doi:10.1186/ar4682.

13. Dimitrov S, Lange T, Nohroudi K, Born J. Number and function of circulating human antigen presenting cells regulated by sleep. Sleep. 2007;30(4):401-411.

14. Dimitrov S, Lange T, Tieken S, Fehm HL, Born J. Sleep associated regulation of $\mathrm{T}$ helper $1 / \mathrm{T}$ helper 2 cytokine balance in humans. Brain Behav Immun. 2004; 18(4):341-348. doi:10.1016/j.bbi.2003.08.004.

15. Kok VC, Tsai HJ, Su CF, Lee CK. The risks for ovarian, endometrial, breast, colorectal, and other cancers in women with newly diagnosed endometriosis or adenomyosis: a population-based study. Int $\mathrm{J}$ Gynecol Cancer. 2015;25(6):968-976. doi:10.1097/IGC.0000000000000454.

16. Kok VC, Horng JT, Huang HK, Chao TM, Hong YF. Regular inhaled corticosteroids in adult-onset asthma and the risk for future cancer: a population-based cohort study with proper person-time analysis. Ther Clin Risk Manag. 2015;11:489-499. doi:10.2147/TCRM.S80793.

17. Kok VC, Horng JT, Huang JL, Yeh KW, Gau JJ, Chang CW, et al. Population-based cohort study on the risk of malignancy in East Asian children with juvenile idiopathic arthritis. BMC Cancer. 2014;14:634. doi:10.1186/1471-2407-14-634.
18. Kok VC, Horng JT, Chang WS, Hong YF, Chang TH. Allopurinol therapy in gout patients does not associate with beneficial cardiovascular outcomes: a population-based matched-cohort study. PLoS One. 2014;9(6):e99102. doi:10.1371/journal.pone.0099102.

19. Kok VC, Horng JT, Lin HL, Chen YC, Chen YJ, Cheng KF. Gout and subsequent increased risk of cardiovascular mortality in nondiabetics aged 50 and above: a population-based cohort study in Taiwan. BMC Cardiovasc Disord. 2012;12:108. doi:10.1186/14712261-12-108.

20. Hsieh V, Yang Y-H, Liou S-H, Li C-Y, Wu T-N. Influence of socioeconomic status on the association between prenatal periodontal care and preterm low birthweight delivery: a population-based case-control study. J Res Pract Dent. 2014;1-15. doi: 10.5171/2014.469747

21. Benjamini Y, Hochberg Y. Controlling the false discovery rate: a practical and powerful approach in multiple testings. J R Stat Soc Ser B. 1995;57(1):289-300.

22. Glickman ME, Rao SR, Schultz MR. False discovery rate control is a recommended alternative to Bonferroni-type adjustments in health studies. J Clin Epidemiol. 2014;67(8):850-857. doi:10.1016/j.jclinepi. 2014.03.012.

23. Gomez-Gonzalez B, Dominguez-Salazar E, Hurtado-Alvarado G, Esqueda-Leon E, Santana-Miranda R, Rojas-Zamorano JA, et al. Role of sleep in the regulation of the immune system and the pituitary hormones. Ann N Y Acad Sci. 2012;1261:97-106. doi:10.1111/j.17496632.2012.06616.x.

24. Zeng QY, Chen R, Darmawan J, Xiao ZY, Chen SB, Wigley R, et al. Rheumatic diseases in China. Arthritis Res Ther. 2008;10(1):R17. doi:10. 1186/ar2368.

25. Budhiraja R, Roth T, Hudgel DW, Budhiraja P, Drake CL. Prevalence and polysomnographic correlates of insomnia comorbid with medical disorders. Sleep. 2011;34(7):859-867. doi:10.5665/SLEEP.1114.

26. Maslinska M, Przygodzka M, Kwiatkowska B, Sikorska-Siudek K. Sjogren's syndrome: still not fully understood disease. Rheumatol Int. 2015;35(2):233-241. doi:10.1007/s00296-014-3072-5.

27. Aho V, Ollila HM, Rantanen V, Kronholm E, Surakka I, van Leeuwen WM, et al. Partial sleep restriction activates immune response-related gene expression pathways: experimental and epidemiological studies in humans. PLoS One. 2013;8(10):e77184. doi:10.1371/journal.pone. 0077184.

28. Roth T, Berglund P, Shahly V, Shillington AC, Stephenson JJ, Kessler RC. Middle-of-the-night hypnotic use in a large national health plan. J Clin Sleep Med. 2013;9(7):661-668. doi:10.5664/jcsm.2832. 